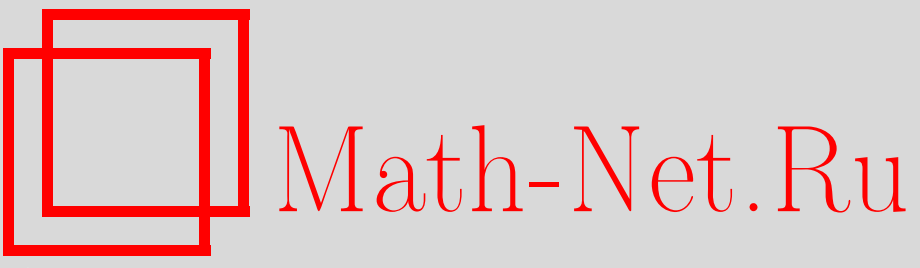

E. Lisovskaya, S. Moiseeva, M. Pagano, V. Potatueva, Study of the MMPP/GI/ $\infty$ queueing system with random customers' capacities, Inform. Primen., 2017, Volume 11, Issue 4, 109-117

DOI: https://doi.org/10.14357/19922264170414

Use of the all-Russian mathematical portal Math-Net.Ru implies that you have read and agreed to these terms of use

http: //www. mathnet.ru/eng/agreement

Download details:

IP: 131.114 .248 .224

March 29, 2019, 19:38:59 


\title{
STUDY OF THE MMPP/GI/ $\infty$ QUEUEING SYSTEM WITH RANDOM CUSTOMERS' CAPACITIES
}

\author{
E. Lisovskaya ${ }^{1}$, S. Moiseeva ${ }^{1}$, M. Pagano ${ }^{2}$, and V. Potatueva ${ }^{1}$
}

\begin{abstract}
A queueing system with an infinite number of servers is considered. Customers arrive in the system according to a Markov Modulated Poisson Process (MMPP). Each customer carries a random quantity of work (capacity of the customer). In this study, service time does not depend on the customers' capacities; the latter are used just to fix some additional features of the system's evolution. It is shown that the joint probability distribution of the customers' number and total capacities in the system is two-dimensional Gaussian under the asymptotic condition of an infinitely growing service time. Simulation results allow determining the applicability area of the asymptotic result.
\end{abstract}

Keywords: infinite-server queueing system; random capacity of customers; Markov Modulated Poisson Process

DOI: $10.14357 / 19922264170414$

\section{Introduction}

Queueing systems represent a powerful mathematical tool for investigating the performance of a wide variety of real-life systems, ranging from telecommunication networks to financial markets, from computer architectures to supply chain management and airplane traffic control, just to cite a few. Analytical tractability of the corresponding models strongly depends on the nature of the underlying processes (Poisson arrivals have many nice features that strongly simplify the analysis) and on the system geometry.

Although physical resources are always finite, quite often it is easier to study queueing systems in which the corresponding parameters assume infinite values. For instance, the overflow probability is often used as an upper bound for the loss probability in finite-buffer queues and, indeed, asymptotic results are available even for strongly non-Markovian systems [1]. Moreover, infinite-server queueing systems may be applicable in case of models with a limited number of server devices as described in [2].

In this work, an infinite-server queueing system, fed by non-Poisson arrivals with random customers' capacities, is considered. Queues with random customers' capacities are useful for analysis and design issues in high-performance computer and communication systems, in which service time and customer volume are the independent quantities (see $[3,4]$ and references therein). For instance, in [3], performance analysis of LTE (Long Term Evolution) networks is carried out in terms of flow-level dynamics and the amount of required radio resources does not depend on the duration of the flow. Such queues are also important in modeling devices, where it is necessary to calculate a sufficient volume of buffer for data storing $[5,6]$. The results for single-server queues with limited buffer and LIFO (last in, first out) service discipline were presented in [7], where algorithms for the calculation of stationary characteristics were derived.

A new trend in the study of queueing systems is the analysis of the systems with non-Poisson arrivals and nonexponential service time. So, in the works [2,8-11], queues with renewal arrivals, Markovian Arrival processes (MAP), and MMPP are studied under various asymptotic conditions. The main contribution of this paper consists in extending such analysis, focusing on the properties of the bidimensional process describing the number of customers and the total capacity in the system when an infinite-server queue is fed by MMPP arrivals with random capacities and nonexponential service time distribution.

\section{Matematical Model}

Consider a queue with infinite number of servers (Fig. 1) and assume that customers arrive according to an MMPP. The input process is defined by its generator matrix $\mathbf{Q}=\left\|q_{i j}\right\|$ of size $K \times K$ and the conditional rates $\lambda_{1}, \ldots, \lambda_{K}$, typically composed into the diagonal matrix $\boldsymbol{\Lambda}=\operatorname{diag}\left\{\lambda_{1}, \ldots, \lambda_{K}\right\}$. Denote the underlying

\footnotetext{
${ }^{1}$ Tomsk State University, 36 Lenin ave., Tomsk 634050, Russian Federation

${ }^{2}$ University of Pisa, 16 Via Caruso, Pisa 56122, Italy
} 


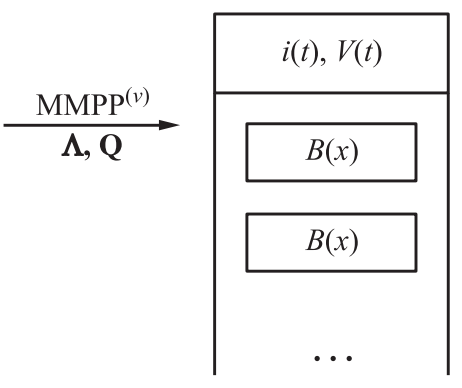

Figure 1 Queue MMPP/GI/ $\infty$ with random customers' capacities

Markov chain of the MMPP as $k(t) \in 1,2, \ldots, K$. Let each customer has some random capacity $v>0$ with distribution function $G(y)$. An arriving customer instantly occupies a server in the system and its service time has distribution function $B(x)$; when the service is completed, the customer leaves the system. Customers' capacities and service times are mutually independent and do not dependent on the epochs of customers' arrivals.

Denote by $i(t)$ and $V(t)$ the number of customers in the system at time $t$ and their total capacity, respectively. Let us obtain the probabilistic characteristics of twodimensional process $\{i(t), V(t)\}$. This process is not Markovian; therefore, the dynamic screening method has been used for its investigation.

Consider two time axes that are numbered as 0 and 1 (Fig. 2). Let axis 0 shows the epochs of customers' arrivals, while axis 1 corresponds to the screened process.

Let us introduce a function $S(t)$ (dynamic probability) that satisfies the condition $0 \leq S(t) \leq 1$. Let us assume that a customer, arriving in the system at time $t$, is screened to axis 1 with probability $S(t)$, and not screened with probability $1-S(t)$.

Let the system be empty at moment $t_{0}$ and let us fix some arbitrary moment $T$ in the future. $S(t)$ represents the probability that a customer arriving at time $t$ will be serviced in the system by the moment $T$. It is easy to show [11] that $S(t)=1-B(T-t)$ for $t_{0} \leq t \leq T$.

Denote by $n(t)$ and $W(t)$ the number of arrivals screened before the moment $t$ on axis 1 and their total

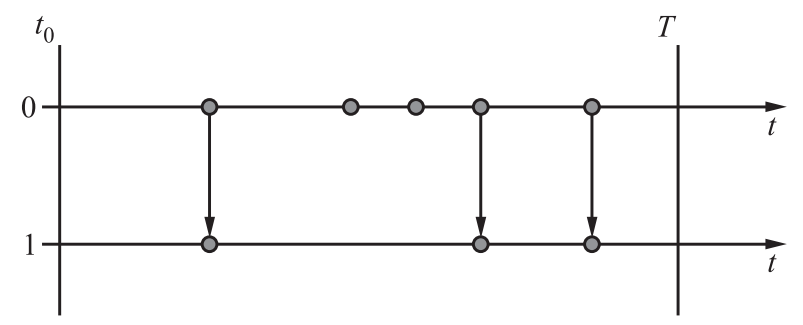

Figure 2 Screening of the customers' arrivals capacity, respectively. As it is shown in [9], the probability distribution of the number of customers in the system at the moment $T$ coincides with the probability distribution of the number of screened arrivals on the axis:

$$
P\{i(T)=m\}=P\{n(T)=m\}
$$

for all $m=0,1,2, \ldots$ It is easy to prove the same property for the extended process $\{i(t), V(t)\}$ :

$$
\begin{aligned}
P\{i(T)=m, V(T) & <z\} \\
& =P\{n(T)=m, W(T)<z\}
\end{aligned}
$$

for all $m=0,1,2, \ldots$ and $z \geq 0$. Let us use Eqs. (1) for the investigation of the process $\{i(t), V(t)\}$ via the analysis of the process $\{n(t), W(t)\}$.

\section{Kolmogorov Differential Equations}

Let us consider the three-dimensional Markovian process $\{k(t), n(t), W(t)\}$. Denoting the probability distribution of this process by $P(k, n, w, t)=P\{k(t)$ $=k, n(t)=n, W(t)<w\}$ and taking into account the formula of total probability, one can write the following system of Kolmogorov differential equations:

$$
\begin{aligned}
\frac{\partial P(k, n, w, t)}{\partial t} & =\lambda_{k} S(t)\left[\int_{0}^{z} P(k, n-1, w-y, t) d G(y)\right. \\
& -P(k, n, w, t)]+\sum_{v} P(\nu, n, w, t) q_{\nu k}
\end{aligned}
$$

for $k=1, \ldots, K ; n=0,1,2, \ldots ; w>0$.

Let us introduce the partial characteristic function:

$$
h\left(k, u_{1}, u_{2}, t\right)=\sum_{n=0}^{\infty} e^{j u_{1} n} \int_{0}^{\infty} e^{j u_{2} w} P(k, n, d w, t)
$$

where $j=\sqrt{-1}$ is the imaginary unit. Then, one can write the following equations:

$$
\begin{aligned}
& \frac{\partial h\left(k, u_{1}, u_{2}, t\right)}{\partial t} \\
& =h\left(k, u_{1}, u_{2}, t\right) \lambda_{k} S(t) \\
& \left(e^{j u_{1}} G^{*}\left(u_{2}\right)-1\right) \\
& +\sum_{\nu} h(\nu, n, w, t) q_{\nu k}
\end{aligned}
$$

for $k=1, \ldots, K$ where $G^{*}(u)=\int_{0}^{\infty} e^{j u y} d G(y)$.

Let us rewrite this system in the matrix form:

$$
\begin{aligned}
& \frac{\partial \mathbf{h}\left(u_{1}, u_{2}, t\right)}{\partial t} \\
& =\mathbf{h}\left(u_{1}, u_{2}, t\right)\left[\boldsymbol{\Lambda} S(t)\left(e^{j u_{1}} G^{*}\left(u_{2}\right)-1\right)+\mathbf{Q}\right]
\end{aligned}
$$


with the initial condition

$$
\mathbf{h}\left(u_{1}, u_{2}, t_{0}\right)=\mathbf{r}
$$

where

$$
\mathbf{h}\left(u_{1}, u_{2}, t\right)=\left[h\left(1, u_{1}, u_{2}, t\right), \ldots, h\left(K, u_{1}, u_{2}, t\right)\right]
$$

and $\mathbf{r}=[r(1), \ldots, r(K)]$ represents the stationary distribution of the underlying Markov chain, i. e., vector $\mathbf{r}$ satisfies the following linear system:

$$
\left.\begin{array}{l}
\mathbf{r Q}=\mathbf{0} \\
\mathbf{r e}=1
\end{array}\right\}
$$

where $\mathbf{e}$ is the column vector with all entries equal to 1 .

\section{Asymptotic Analysis}

In general, the exact solution of Equation (2) is not available, but it may be found under asymptotic conditions. In this paper, the case of infinitely growing service time is considered.

Denote by

$$
b_{1}=\int_{0}^{\infty} x d B(x)=\int_{0}^{\infty}(1-B(x)) d x
$$

the mean service time; then, the asymptotic condition is $b_{1} \rightarrow \infty$.

Let us solve Problem (2)-(3) under such asymptotic condition and we obtain approximate solutions with different order of accuracy, named as "first-order asymptotic" $\mathbf{h}\left(u_{1}, u_{2}, t\right) \approx \mathbf{h}^{(1)}\left(u_{1}, u_{2}, t\right)$ and "second-order asymptotic" $\mathbf{h}\left(u_{1}, u_{2}, t\right) \approx \mathbf{h}^{(2)}\left(u_{1}, u_{2}, t\right)$.

\subsection{First-order asymptotic analysis}

Let us formulate and prove the following statement.

Lemma. The first-order asymptotic characteristic function of the probability distribution of the process $\{k(t), n(t), W(t)\}$ has the form:

$\mathbf{h}^{(1)}\left(u_{1}, u_{2}, t\right)=\mathbf{r} \exp \left\{\left(j u_{1} \kappa_{1}+j u_{2} \kappa_{1} a_{1}\right) \int_{t_{0}}^{t} S(v) d v\right\}$

where $\kappa_{1}=\mathbf{r} \Lambda \mathbf{e}$ and $a_{1}=\int_{0}^{\infty} y d G(y)$ is the mean customer capacity.

Pro of. By performing the substitutions

$$
\begin{gathered}
\varepsilon=\frac{1}{b_{1}} ; \quad \varepsilon t=\tau ; \quad \varepsilon t_{0}=\tau_{0} ; \\
u_{1}=\varepsilon x_{1} ; u_{2}=\varepsilon x_{2} ; \quad S(t)=S_{1}(\tau) ; \\
\mathbf{h}\left(u_{1}, u_{2}, t\right)=\mathbf{f}_{1}\left(x_{1}, x_{2}, \tau, \varepsilon\right)
\end{gathered}
$$

in expressions (2) and (3), one obtains

$$
\varepsilon \frac{\partial \mathbf{f}_{1}\left(x_{1}, x_{2}, \tau, \varepsilon\right)}{\partial \tau}
$$

$$
=\mathbf{f}_{1}\left(x_{1}, x_{2}, \tau, \varepsilon\right)\left[\boldsymbol{\Lambda} S_{1}(\tau)\left(e^{j \varepsilon x_{1}} G^{*}\left(\varepsilon x_{2}\right)-1\right)+\mathbf{Q}\right]
$$

with the initial condition

$$
\mathbf{f}_{1}\left(x_{1}, x_{2}, \tau_{0}, \varepsilon\right)=\mathbf{r} .
$$

Let us find the asymptotic solution of Problem (5)(6) $\mathbf{f}_{1}\left(x_{1}, x_{2}, \tau\right)=\lim _{\varepsilon \rightarrow 0} \mathbf{f}_{1}\left(x_{1}, x_{2}, \tau, \varepsilon\right)$ in two steps.

Step 1. Let $\varepsilon \rightarrow 0$ in (5)-(6); then, one obtains the following system of equations:

$$
\left\{\begin{array}{l}
\mathbf{f}_{1}\left(x_{1}, x_{2}, \tau\right) \mathbf{Q}=\mathbf{0} \\
\mathbf{f}_{1}\left(x_{1}, x_{2}, \tau_{0}\right)=\mathbf{r}
\end{array}\right.
$$

Taking into account (4), one can conclude that $\mathbf{f}_{1}\left(x_{1}, x_{2}, \tau\right)$ can be expressed as

$$
\mathbf{f}_{1}\left(x_{1}, x_{2}, \tau\right)=\mathbf{r} \Phi_{1}\left(x_{1}, x_{2}, \tau\right)
$$

where $\Phi_{1}\left(x_{1}, x_{2}, \tau\right)$ is some scalar function which satisfies the condition

$$
\Phi_{1}\left(x_{1}, x_{2}, \tau_{0}\right)=1 .
$$

Step 2. Let us multiply (5) by vector e, substitute (7), divide the result by $\varepsilon$, and perform the asymptotic transition $\varepsilon \rightarrow 0$. Then, taking into account that $\mathbf{Q e}=\mathbf{0}$ and re $=1$, one obtains the following differential equation for the function $\Phi_{1}\left(x_{1}, x_{2}, \tau\right)$ :

$$
\begin{aligned}
& \frac{\partial \Phi_{1}\left(x_{1}, x_{2}, \tau\right)}{\partial \tau} \\
& \quad=\Phi_{1}\left(x_{1}, x_{2}, \tau\right) S_{1}(\tau)\left(j x_{1} \kappa_{1}+j x_{2} \kappa_{1} a_{1}\right) .
\end{aligned}
$$

The solution of Problem (8)-(9) is as follows:

$$
\Phi_{1}\left(x_{1}, x_{2}, \tau\right)=\exp \left\{\left(j x_{1} \kappa_{1}+j x_{2} \kappa_{1} a_{1}\right) \int_{\tau_{0}}^{\tau} S_{1}(v) d v\right\} .
$$

Substituting this expression into (7), one obtains

$$
\mathbf{f}_{1}\left(x_{1}, x_{2}, \tau\right)=\mathbf{r} \exp \left\{\left(j x_{1} \kappa_{1}+j x_{2} \kappa_{1} a_{1}\right) \int_{\tau_{0}}^{\tau} S_{1}(v) d v\right\} .
$$

Therefore, one can write

$$
\begin{gathered}
\mathbf{h}^{(1)}\left(u_{1}, u_{2}, t\right)=\mathbf{f}_{1}\left(x_{1}, x_{2}, \tau, \varepsilon\right) \approx \mathbf{f}_{1}\left(x_{1}, x_{2}, \tau\right) \\
=\mathbf{r} \exp \left\{\left(j x_{1} \kappa_{1}+j x_{2} \kappa_{1} a_{1}\right) \int_{\tau_{0}}^{\tau} S_{1}(v) d v\right\} \\
=\mathbf{r} \exp \left\{\left(j u_{1} \kappa_{1}+j u_{2} \kappa_{1} a_{1}\right) \int_{t_{0}}^{t} S(v) d v\right\} .
\end{gathered}
$$

Thus, the proof is complete. 


\subsection{Second-order asymptotic analysis}

The main result is the following theorem.

Theorem. The second-order asymptotic characteristic function of the probability distribution of the process $\{k(t), n(t), W(t)\}$ has the form:

$$
\begin{gathered}
\mathbf{h}^{(2)}\left(u_{1}, u_{2}, t\right) \\
=\mathbf{r} \exp \left\{\left(j u_{1} \kappa_{1}+j u_{2} \kappa_{1} a_{1}\right) \int_{t_{0}}^{t} S(v) d v\right. \\
+\frac{\left(j u_{1}\right)^{2}}{2}\left(\kappa_{1} \int_{t_{0}}^{t} S(v) d v+\kappa_{2} \int_{t_{0}}^{t} S^{2}(v) d v\right) \\
+\frac{\left(j u_{2}\right)^{2}}{2}\left(\kappa_{1} a_{2} \int_{t_{0}}^{t} S(v) d v+\kappa_{2} a_{1}^{2} \int_{t_{0}}^{t} S^{2}(v) d v\right) \\
\left.+j u_{1} j u_{2}\left(\kappa_{1} a_{1} \int_{t_{0}}^{t} S(v) d v+\kappa_{2} a_{1} \int_{t_{0}}^{t} S^{2}(v) d v\right)\right\}
\end{gathered}
$$

where $\kappa_{2}=2 \mathbf{g}\left(\boldsymbol{\Lambda}-\kappa_{1} \mathbf{I}\right) \mathbf{e} ; a_{2}=\int_{0}^{\infty} y^{2} d G(y)$; and the row vector $\mathrm{g}$ satisfies the linear matrix system

$$
\left\{\begin{array}{l}
\mathbf{g Q}=\mathbf{r}\left(\kappa_{1} \mathbf{I}-\mathbf{\Lambda}\right) ; \\
\mathbf{g e}=\text { const } .
\end{array}\right.
$$

Proof. Let $\mathbf{h}_{2}\left(x_{1}, x_{2}, t\right)$ be a vector function that satisfies the equation:

$$
\begin{aligned}
& \mathbf{h}\left(u_{1}, u_{2}, t\right)=\mathbf{h}_{2}\left(u_{1}, u_{2}, t\right) \\
& \times \exp \left\{\left(j u_{1} \kappa_{1}+j u_{2} \kappa_{1} a_{1}\right) \int_{t_{0}}^{t} S(v) d v\right\} .
\end{aligned}
$$

Substituting this expression into (2) and (3), one obtains the following problem:

$$
\begin{aligned}
& \frac{\partial \mathbf{h}_{2}\left(u_{1}, u_{2}, t\right)}{\partial t} \\
& \quad=\mathbf{h}_{2}\left(u_{1}, u_{2}, t\right)\left[\left(e^{j u_{1}} G^{*}\left(u_{2}\right)-1\right) S(t) \mathbf{\Lambda}\right. \\
& \left.\quad-\left(j u_{1} \kappa_{1}+j u_{2} \kappa_{1} a_{1}\right) S(t) \mathbf{I}+\mathbf{Q}\right]
\end{aligned}
$$

with the initial condition

$$
\mathbf{h}_{2}\left(u_{1}, u_{2}, t_{0}\right)=\mathbf{r}
$$

where $\mathbf{I}$ is the identity matrix.

Let us make the substitutions:

$$
\left.\begin{array}{c}
\varepsilon^{2}=\frac{1}{b_{1}} ; \quad \varepsilon^{2} t=\tau ; \quad \varepsilon^{2} t_{0}=\tau_{0} ; \\
u_{1}=\varepsilon x_{1} ; \quad u_{2}=\varepsilon x_{2} ; \quad S(t)=S_{1}(t) ; \\
\mathbf{h}_{2}\left(u_{1}, u_{2}, t\right)=\mathbf{f}_{2}\left(x_{1}, x_{2}, \tau, \varepsilon\right) .
\end{array}\right\}
$$

Using these notations, Problem (11)-(12) can be rewritten in the form

$$
\begin{aligned}
\varepsilon^{2} & \frac{\partial \mathbf{f}_{2}\left(x_{1}, x_{2}, \tau, \varepsilon\right)}{\partial \tau} \\
= & \mathbf{f}_{2}\left(x_{1}, x_{2}, \tau, \varepsilon\right)\left[\boldsymbol{\Lambda} S_{1}(\tau)\left(e^{j \varepsilon x_{1}} G^{*}\left(\varepsilon x_{2}\right)-1\right)\right. \\
& \left.\quad-\left(j \varepsilon \kappa_{1} x_{1}+j \varepsilon \kappa_{1} x_{2} a_{1}\right) S_{1}(\tau) \mathbf{I}+\mathbf{Q}\right]
\end{aligned}
$$

with the initial condition

$$
\mathbf{f}_{2}\left(x_{1}, x_{2}, \tau_{0}, \varepsilon\right)=\mathbf{r} .
$$

Let us find the asymptotic solution of this problem $\mathbf{f}_{2}\left(x_{1}, x_{2}, \tau\right)=\lim _{\varepsilon \rightarrow 0} \mathbf{f}_{2}\left(x_{1}, x_{2}, \tau, \varepsilon\right)$ in three steps.

Step 1. Letting $\varepsilon \rightarrow 0$ in (14)-(15), one obtains the following system of equations:

$$
\left\{\begin{array}{l}
\mathbf{f}_{2}\left(x_{1}, x_{2}, \tau\right) \mathbf{Q}=\mathbf{0} ; \\
\mathbf{f}_{2}\left(x_{1}, x_{2}, \tau_{0}\right)=\mathbf{r} .
\end{array}\right.
$$

Therefore, taking into account (4), one can write:

$$
\mathbf{f}_{2}\left(x_{1}, x_{2}, \tau\right)=\mathbf{r} \Phi_{2}\left(x_{1}, x_{2}, \tau\right)
$$

where $\Phi_{2}\left(x_{1}, x_{2}, \tau\right)$ is some scalar function which satisfies the condition

$$
\Phi_{2}\left(x_{1}, x_{2}, \tau_{0}\right)=1 .
$$

Step 2. Using (16), the function $\mathbf{f}_{2}\left(x_{1}, x_{2}, \tau\right)$ can be represented in the expansion form:

$$
\begin{aligned}
\mathbf{f}_{2}\left(x_{1}, x_{2}, \tau, \varepsilon\right) & \\
=\Phi_{2}\left(x_{1}, x_{2}, \tau\right)\left[\mathbf{r}+\mathbf{g} S_{1}(\tau)\left(j \varepsilon x_{1}\right.\right. & \left.\left.+j \varepsilon x_{2} a_{1}\right)\right] \\
& +\mathbf{O}\left(\varepsilon^{2}\right)
\end{aligned}
$$

where $\mathbf{g}$ is the row vector that satisfies the condition ge $=$ const and $\mathbf{O}\left(\varepsilon^{2}\right)$ is the row vector of the secondorder infinitesimals. Let us use substitution (18) and the expansion

$$
e^{j \varepsilon x}=1+j \varepsilon x+O\left(\varepsilon^{2}\right)
$$

in Eq. (14). Taking into account (4) and making the transition $\varepsilon \rightarrow 0$, one obtains the following matrix equation for the vector $\mathrm{g}$ :

$$
\mathbf{g} \mathbf{Q}=\mathbf{r}\left(\kappa_{1} \mathbf{I}-\mathbf{\Lambda}\right) .
$$

Step 3. Let us multiply Eq. (14) by vector e and use expression (18) and the second-order expansion:

$$
e^{j \varepsilon x}=1+j \varepsilon x+\frac{(j \varepsilon x)^{2}}{2}+O\left(\varepsilon^{3}\right) .
$$


After some transformations, using the notation

$$
\kappa_{2}=2 \mathbf{g}\left(\boldsymbol{\Lambda}-\kappa_{1} \mathbf{I}\right) \mathbf{e},
$$

one obtains the following differential equation for the function $\Phi_{2}\left(x_{1}, x_{2}, \tau\right)$ :

$$
\begin{aligned}
& \frac{\partial \Phi_{2}\left(x_{1}, x_{2}, \tau\right)}{\partial \tau} \\
& =\Phi_{2}\left(x_{1}, x_{2}, \tau\right)\left[\frac{\left(j x_{1}\right)^{2}}{2}\left(\kappa_{1} S_{1}(\tau)+\kappa_{2} S_{1}^{2}(\tau)\right)\right. \\
& \quad+\frac{\left(j x_{2}\right)^{2}}{2}\left(\kappa_{1} a_{2} S_{1}(\tau)+\kappa_{2} a_{1}^{2} S_{1}^{2}(\tau)\right) \\
& \left.\quad+j x_{1} j x_{2}\left(\kappa_{1} a_{1} S_{1}(\tau)+\kappa_{2} a_{1} S_{1}^{2}(\tau)\right)\right] .
\end{aligned}
$$

The solution of this equation with initial condition (17) is as follows:

$$
\begin{aligned}
& \Phi_{2}\left(x_{1}, x_{2}, \tau\right) \\
& =\exp \left\{\frac{\left(j x_{1}\right)^{2}}{2}\left(\kappa_{1} \int_{\tau_{0}}^{\tau} S_{1}(v) d v+\kappa_{2} \int_{\tau_{0}}^{\tau} S_{1}^{2}(v) d v\right)\right. \\
& +\frac{\left(j x_{2}\right)^{2}}{2}\left(\kappa_{1} a_{2} \int_{\tau_{0}}^{\tau} S_{1}(v) d v+\kappa_{2} a_{1}^{2} \int_{\tau_{0}}^{\tau} S_{1}^{2}(v) d v\right) \\
& \left.+j x_{1} j x_{2}\left(\kappa_{1} a_{1} \int_{\tau_{0}}^{\tau} S_{1}(v) d v+\kappa_{2} a_{1} \int_{\tau_{0}}^{\tau_{1}} S_{1}^{2}(v) d v\right)\right\}
\end{aligned}
$$

Substituting this expression in formula (16) and performing the substitutions that are inverse to (13) and (10), one obtains

$$
\begin{array}{r}
\mathbf{h}^{(2)}\left(u_{1}, u_{2}, t\right) \\
=\mathbf{r} \exp \left\{\left(j u_{1} \kappa_{1}+j u_{2} \kappa_{1} a_{1}\right) \int_{t_{0}}^{t} S(v) d v\right. \\
+\frac{\left(j u_{1}\right)^{2}}{2}\left(\kappa_{1} \int_{t_{0}}^{t} S(v) d v+\kappa_{2} \int_{t_{0}}^{t} S^{2}(v) d v\right) \\
+\frac{\left(j u_{2}\right)^{2}}{2}\left(\kappa_{1} a_{2} \int_{t_{0}}^{t} S(v) d v+\kappa_{2} a_{1}^{2} \int_{t_{0}}^{t} S^{2}(v) d v\right) \\
\left.+j u_{1} j u_{2}\left(\kappa_{1} a_{1} \int_{t_{0}}^{t} S(v) d v+\kappa_{2} a_{1} \int_{t_{0}}^{2} S^{2}(v) d v\right)\right\}
\end{array}
$$

for the asymptotic characteristic function of the process $\{k(t), n(t), W(t)\}$. The proof is complete.
Corollary. Assuming $t=T$ and $t_{0} \rightarrow-\infty$ and using Eqs. (1), one obtains the steady-state characteristic function of the process under study $\{i(t), V(t)\}$ :

$$
\begin{aligned}
& h\left(u_{1}, u_{2}\right)=\exp \left\{\left(j u_{1} \kappa_{1} b_{1}+j u_{2} \kappa_{1} a_{1} b_{1}\right)\right. \\
& +\frac{\left(j u_{1}\right)^{2}}{2}\left(\kappa_{1} b_{1}+\kappa_{2} b_{2}\right)+\frac{\left(j u_{2}\right)^{2}}{2}\left(\kappa_{1} a_{2} b_{1}+\kappa_{2} a_{1}^{2} b_{2}\right) \\
& \left.+j u_{1} j u_{2}\left(\kappa_{1} a_{1} b_{1}+\kappa_{2} a_{1} b_{2}\right)\right\}
\end{aligned}
$$

where

$$
b_{1}=\int_{0}^{\infty}(1-B(v)) d v ; \quad b_{2}=\int_{0}^{\infty}(1-B(v))^{2} d v .
$$

From the form of the characteristic function (19), it is clear that the probability distribution of the twodimensional process $\{i(t), V(t)\}$ is asymptotically Gaussian with vector of means

$$
\mathbf{a}=\left[\kappa_{1} b_{1} \kappa_{1} a_{1} b_{1}\right]
$$

and covariance matrix

$$
\begin{aligned}
\mathbf{K}=\left[\begin{array}{cc}
\sigma_{1}^{2} & K_{12} \\
K_{12} & \sigma_{2}^{2}
\end{array}\right] \\
\quad=\left[\begin{array}{cc}
\kappa_{1} b_{1}+\kappa_{2} b_{2} & \kappa_{1} a_{1} b_{1}+\kappa_{2} a_{1} b_{2} \\
\kappa_{1} a_{1} b_{1}+\kappa_{2} a_{1} b_{2} & \kappa_{1} a_{2} b_{1}+\kappa_{2} a_{1}^{2} b_{2}
\end{array}\right] .
\end{aligned}
$$

Therefore, the correlation coefficient is given by

$$
r=\frac{K_{12}}{\sigma_{1} \sigma_{2}}=\frac{\kappa_{1} a_{1} b_{1}+\kappa_{2} a_{1} b_{2}}{\sqrt{\kappa_{1} b_{1}+\kappa_{2} b_{2}} \sqrt{\kappa_{1} a_{2} b_{1}+\kappa_{2} a_{1}^{2} b_{2}}} .
$$

\section{Numerical Example}

Result (19) is obtained under the asymptotic condition $b_{1} \rightarrow \infty$. Therefore, it may be used just as an approximation when $b_{1}$ is large enough. To test its practical applicability, the present authors considered several numerical examples, varying all the system parameters (including the distributions of the service time and of the customer capacity). Since all the different simulation sets led to similar results, for sake of brevity, in the following, just one of them is discussed in detail. In particular, let us assume that the input MMPP is characterized by the matrices:

$$
\mathbf{Q}=\left[\begin{array}{rrr}
-0.8 & 0.4 & 0.4 \\
0.3 & -0.6 & 0.3 \\
0.4 & 0.4 & -0.8
\end{array}\right]
$$

and

$$
\boldsymbol{\Lambda}=\left[\begin{array}{ccc}
0.5 & 0 & 0 \\
0 & 1 & 0 \\
0 & 0 & 1.5
\end{array}\right]
$$


Table 1 Kolmogorov distances between simulation results and asymptotic values for the number of customers in the system

\begin{tabular}{rc}
\hline$N$ & $\Delta$ \\
\hline 1 & 0.265 \\
10 & 0.039 \\
15 & 0.032 \\
20 & $\mathbf{0 . 0 2 7}$ \\
25 & $\mathbf{0 . 0 2 5}$ \\
50 & $\mathbf{0 . 0 1 7}$ \\
100 & $\mathbf{0 . 0 1 2}$ \\
\hline
\end{tabular}

Table 2 Kolmogorov distances between simulation results and asymptotic values for the total capacity in the system

\begin{tabular}{rc}
\hline$N$ & $\Delta$ \\
\hline 1 & 0.355 \\
10 & 0.033 \\
15 & $\mathbf{0 . 0 2 5}$ \\
20 & $\mathbf{0 . 0 2 1}$ \\
25 & $\mathbf{0 . 0 1 9}$ \\
50 & $\mathbf{0 . 0 1 3}$ \\
100 & $\mathbf{0 . 0 1 0}$ \\
\hline
\end{tabular}

Hence, the fundamental rate of arrivals is $\kappa_{1}$ $=\mathbf{r} \boldsymbol{\Lambda} \mathbf{e}=1$ customers per time unit. Let us also assume that customers' capacities have uniform distribution in the range $[0 ; 1]$ and service time has gamma distribution with shape and inverse scale parameters $\alpha=1.5$ and $\beta=\alpha / N$, respectively. So, when $N \rightarrow \infty$, one obtains the asymptotic condition of an infinite growing service time $\left(b_{1}=\alpha / \beta=N \rightarrow \infty\right)$.
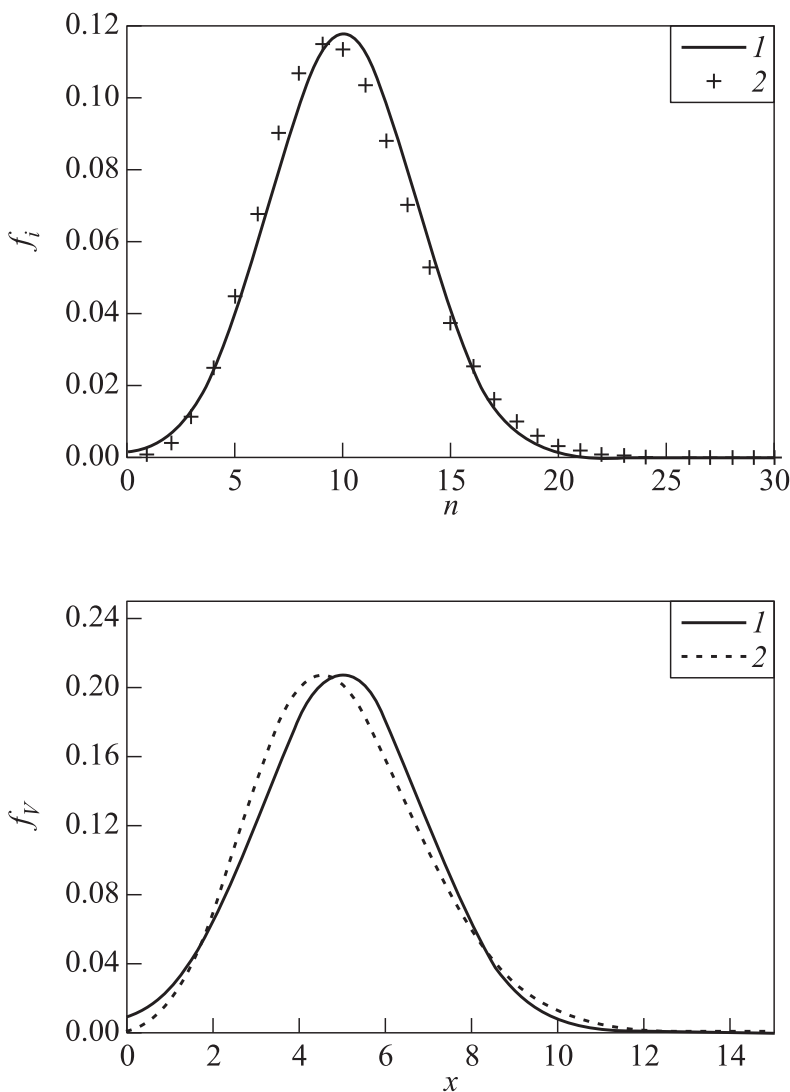

The goal is to find a lower bound of parameter $N$ for the applicability of the approximation (19). To this aim, series of simulation experiments have been carried out for increasing values of $N$ and the asymptotic distributions have been compared with the empiric ones by using the Kolmogorov distance [12, 13]

$$
\Delta=\sup _{x}|F(x)-A(x)|
$$

as an accuracy measure. Here, $F(x)$ is the cumulative distribution function built on the basis of simulation results and $A(x)$ is the Gaussian approximation based on (19).

Let us consider the marginal distributions of the customers' number and the total capacity in the system.

In the first case, the asymptotic values of mean and variance are equal to $N$ and $1.144 N$, respectively, and the corresponding values of the Kolmogorov distance for increasing values of parameter $N$ are presented in Table 1. Similarly, for the total capacity in the system, mean and variance are equal to $0.5 \mathrm{~N}$ and $0.369 \mathrm{~N}$, respectively, and Table 2 shows the Kolmogorov distance.

One can notice that the asymptotic results become more accurate when the parameter $N$ increases. Fig-

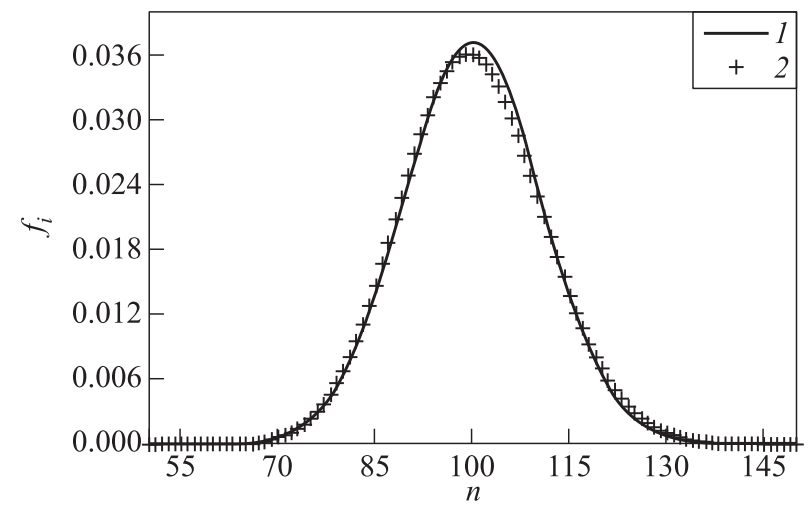

(a)

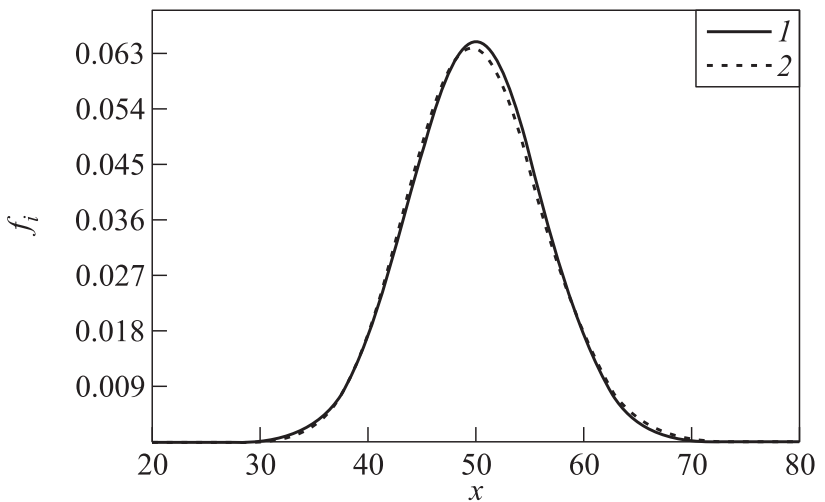

(b)

Figure 3 Distributions of the number of customers $(a)$ and of the total capacity $(b)$ for different values of $N$ : left column $N=10$; right column $-N=100 ; 1-$ theoretical results; and $2-$ simulation 


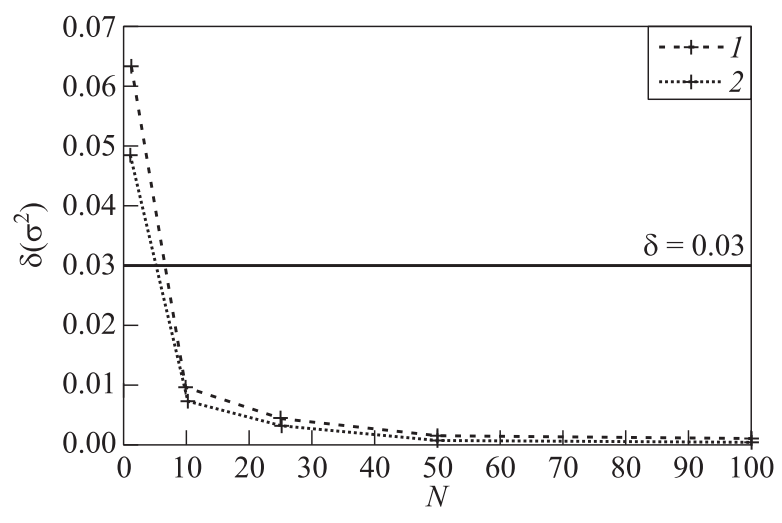

Figure 4 Relative error for the variance of the number of customers $i(t)(1)$ and the total capacity $V(t)(2)$

ure 3 compares the asymptotic approximations with the empirical results for the number of customers and the total capacity in the system.

As typically done in the literature [12], let us suppose that an approximation is applicable if its Kolmogorov distance is less than 0.03 . Hence, one can conclude that the asymptotic results are applicable for values of the parameter $N$ equal to 15 or more (marked by boldface in Tables 1 and 2).

Then, let us compare the asymptotic value of some characteristics of the queueing system with the corresponding empirical characteristics, using the relative error

$$
\delta=\frac{|d-a|}{d}
$$

where $d$ denotes the value constructed on the basis of simulation results and $a$ is obtained from (19).

In more detail, the mean values of the processes $i(t)$ and $V(t)$ are very close (with $\delta<10^{-5}$ for all $N$ ) and the relative errors of the variance decreases with $N$ as shown in Fig. 4.

Finally, Table 3 shows the relative error for the correlation coefficient.

Table 3 Relative error for the correlation coefficient

\begin{tabular}{rr}
\hline$N$ & \multicolumn{1}{c}{$\delta$} \\
\hline 1 & $\mathbf{6 0} \cdot \mathbf{1 0} 0^{-4}$ \\
10 & $\mathbf{1 1} \cdot \mathbf{1 0}^{-4}$ \\
15 & $\mathbf{7} \cdot \mathbf{1 0}^{-4}$ \\
20 & $\mathbf{5} \cdot \mathbf{1 0}^{-4}$ \\
25 & $\mathbf{4} \cdot \mathbf{1 0}^{-4}$ \\
50 & $\mathbf{1} \cdot \mathbf{1 0}^{-4}$ \\
100 & $\mathbf{0 . 8} \cdot \mathbf{1 0}^{-4}$ \\
\hline
\end{tabular}

\section{Concluding Remarks}

In the paper, the queue with MMPP arrivals, infinite number of servers, and nonexponential service time is considered. Moreover, random customers' capacities, independent of their service time, are assumed. The analysis is performed under the asymptotic condition of an infinitely growing service time. It is shown that twodimensional probability distribution of customers' number and total capacity in the system is two-dimensional Gaussian under this asymptotic condition. Numerical results show that asymptotic results have enough accuracy for the marginal distributions of number of customers and of the total capacity in the system when the service rate exceeds the fundamental rate of arrivals by at least 15 times.

\section{Acknowledgments}

This work is supported by the Russian Foundation for Basic research, project 16-31-00292.

\section{References}

1. Mandjes, M. 2007. Large deviations of Gaussian queues. Chichester: Wiley. 340 p.

2. Melikov, A., L. Zadiranova, and A. Moiseev. 2016. Two asymptotic conditions in queue with MMPP arrivals and feedback. Comm. Com. Inf. Sc. 678:231-240. doi: 10.1007/978-3-319-51917-3_21.

3. Naumov, V., K. Samouylov, E. Sopin, and S. Andreev. 2015. Two approaches to analyzing dynamic cellular networks with limited resources. 6th Congress (International) on Ultra Modern Telecommunications and Control Systems and Workshops. St. Petersburg. 485-488. doi: 10.1109/ICUMT.2014.7002149.

4. Morozov, E., L. Potakhina, and O. Tikhonenko. 2016. Regenerative analysis of a system with a random volume of customers. Comm. Com. Inf. Sc. 638:261-272. doi: 10.1007/978-3-319-44615-8_23.

5. Tikhonenko, O. M., and W. Kempa. 2015. Queueing systems with processor sharing and limited memory under control of the AQM mechanism. Automat. Rem. Contr. 76(10):1784-1796. doi: 10.1134/S0005117915100069.

6. Naumov, V. A., K. E. Samuilov, and A. K. Samuilov. 2016. On the total amount of resources occupied by serviced customers. Automat. Rem. Contr. 77(8):1419-1427.

7. Tikhonenko, O. M. 2010. Queueing system with processor sharing and limited resources. Automat. Rem. Contr. 71(5):803-815.

8. Pankratova, E. V., and S. P. Moiseeva. 2014. Queueing system MAP $/ \mathrm{M} / \infty$ with $n$ types of customers. Comm. Com. Inf. Sc. 487:356-366.

9. Moiseev, A., and A. Nazarov. 2016. Tandem of infiniteserver queues with Markovian arrival process. Comm. Com. Inf. Sc. 601:323-333. doi: 10.1007/978-3-319-30843$2 \_34$. 
10. Lisovskaya, E., S. Moiseeva, and M. Pagano. 2016. The total capacity of customers in the infinite-server queue with MMPP arrivals. Comm. Com. Inf. Sc. 678:110-120. doi: 10.1007/978-3-319-51917-3_11.

11. Moiseev, A., and A. Nazarov. 2016. Queueing network $\mathrm{MAP} /(\mathrm{GI} / \infty)^{K}$ with high-rate arrivals. Eur. J. Oper. Res. 254:161-168. doi: 10.1016/j.ejor.2016.04.011.

12. Moiseev, A. N., and M. V. Sinyakov. 2010. Razrabotka ob"ektno-orientirovannoy modeli sistemy imitatsionno- go modelirovaniya protsessov massovogo obsluzhivaniya [Design of object-oriented model for queueing simulation software]. Vestnik Tomskogo gosudarstvennogo universiteta. Upravlenie, vychislitel'naya tekhnika i informatika [Tomsk State University. J. Control Computer Sci.] 1:89-93.

13. Moiseev, A., A. Demin, V. Dorofeev, and V. Sorokin. 2016. Discrete-event approach to simulation of queueing networks. Key Eng. Mater. 685:939-942. doi: 10.4028/www.scientific.net/KEM.685.939.

Received March 16, 2017

\title{
Contributors
}

Lisovskaya Ekaterina Yu. (b. 1992) - PhD student, Department of Probability Theory and Mathematical Statistics, Tomsk State University, 36 Lenin Ave., Tomsk 634050, Russian Federation; ekaterina_lisovs@mail.ru

Moiseeva Svetlana P. (b. 1971) - Doctor of Science in physics and mathematics, associate professor, professor, Department of Probability Theory and Mathematical Statistics, Tomsk State University, 36 Lenin ave., Tomsk 634050, Russian Federation; smoiseeva@mail.ru

Pagano Michele (b. 1968) - PhD in electronics engineering, professor, Department of Information Engineering of University of Pisa, 16 Via Caruso, Pisa 56122, Italy; m.pagano@iet.unipi.it

Potatueva Viktoriya V. (b. 1993) - Master Degree student, Department of Probability Theory and Mathematical Statistics, Tomsk State University, 36 Lenin Ave., Tomsk 634050, Russian Federation; ve-kusik@mail.ru

\section{ИССЛЕДОВАНИЕ СИСТЕМЫ МАССОВОГО ОБСЛУЖИВАНИЯ ММРP/GI/ $\infty$ С ТРЕБОВАНИЯМИ СЛУЧАЙНОГО ОБЪЕМА*}

\author{
Е. Ю. Лисовская ${ }^{1}$, С. П. Моисеева ${ }^{2}$, М. Пагано ${ }^{3}$, В. В. Потатуева ${ }^{4}$ \\ ${ }^{1}$ Национальный исследовательский Томский государственный университет, ekaterina_lisovs@mail.ru \\ ${ }^{2}$ Национальный исследовательский Томский государственный университет, smoiseeva@mail.ru \\ ${ }^{3}$ Университет г. Пиза, Италия, m.pagano@iet.unipi.it \\ ${ }^{4}$ Национальный исследовательский Томский государственный университет, ve-kusik@mail.ru
}

\begin{abstract}
Аннотация: Проведено исследование системы массового обслуживания с неограниченным числом приборов. Заявки поступают в систему в виде марковски-модулированного пуассоновского потока. Каждая заявка несет в себе произвольное количество данных (объем заявки). В этом исследовании время обслуживания не зависит от объема заявок. Показано, что совместное распределение вероятностей числа заявок в системе и их суммарного объема является двумерным гауссовским при асимптотическом условии растущего времени обслуживания. Имитационное моделирование и численные эксперименты позволили определить область применимости асимптотического результата.
\end{abstract}

Ключевые слова: бесконечнолинейная система массового обслуживания; случайный объем заявок; ММРР-поток

DOI: $10.14357 / 19922264170414$

\section{Литература}

1. Mandjes M. Large deviations of Gaussian queues. Chichester: Wiley, 2007. 340 p.

2. Melikov A., Zadiranova L., Moiseev A. Two asymptotic conditions in queue with MMPP arrivals and feedback // Comm. Com. Inf. Sc., 2016. Vol. 678. P. 231-240.

3. Naumov V., Samouylov K., Sopin E., Andreev S. Two approaches to analyzing dynamic cellular networks with limited resources // 6th Congress (International) on U1tra Modern Telecommunications and Control Systems and Workshops. - St. Petersburg, 2015. P. 485-488. doi: 10.1007/978-3-319-44615-8_23.

4. Morozov E., Potakhina L., Tikhonenko O. Regenerative analysis of a system with a random volume of customers // Comm. Com. Inf. Sc., 2016. Vol. 638. P. 261-272. doi: 10.1007/978-3-319-44615-8_23.

* Работа выполнена при частичной поддержке РФФИ (проект 16-31-00292). 
5. Тихоненко О.М., Кемпа В.М. Система с разделением процессора и ограниченным объемом памяти, управляемая механизмом AQM // Автоматика и телемеханика, 2015. № 10. С. 90-105.

6. Наумов В. А., Самуйлов К. Е., Самуйлов А. К. О суммарном объеме ресурсов, занимаемых обслуживаемыми заявками // Автоматика и телемеханика, 2016. № 8. C. $125-132$.

7. Тихоненко О. М. Система обслуживания с разделением процессора и ограниченными ресурсами // Queueing systems with processor sharing and limited resources // Автоматика и телемеханика, 2010. № 5. С. 84-98.

8. Pankratova E.V., Moiseeva S.P. Queueing system $\mathrm{MAP} / \mathrm{M} / \infty$ with $n$ types of customers // Comm. Com. Inf. Sc., 2014. Vol. 487. P. 356-366.

9. Moiseev A., Nazarov A. Tandem of infinite-server queues with Markovian arrival process // Comm. Com. Inf. Sc., 2016. Vol. 601. P. 323-333. doi: 10.1007/978-3-31930843-2_34.
10. Lisovskaya E., Moiseeva S., Pagano M. The total capacity of customers in the infinite-server queue with MMPP arrivals // Comm. Com. Inf. Sc., 2016. Vol. 678. P. 110-120. doi: 10.1007/978-3-319-51917-3_11.

11. Moiseev A., Nazarov A. Queueing network $\mathrm{MAP} /(\mathrm{GI} / \infty)^{K}$ with high-rate arrivals // Eur. J. Oper. Res., 2016. Vol. 254. P. 161-168. doi: 10.1016/ j.ejor.2016.04.011.

12. Моисеев А.Н., Синяков М.В. Разработка объектноориентированной модели системы имитационного моделирования процессов массового обслуживания // Вестник Томского государственного университета. Управление, вычислительная техника и информатика, 2010. № 1. С. 89-93.

13. Moiseev A., Demin A., Dorofeev V., Sorokin V. Discreteevent approach to simulation of queueing networks // Key Eng. Mater., 2016. Vol. 685. P. 939-942. doi: 10.4028/www.scientific.net/KEM.685.939.

Поступила в редакцию 16.03.2017 